\title{
Eine schonende Verbindung von Personen- und Verhältniswahl zum Abbau negativer Stimmgewichte bei Bundestagswahlen
}

\section{Einleitung}

Mit Urteil vom 3. Juli 2008 hat das Bundesverfassungsgericht dem Gesetzgeber aufgegeben, bis zum 30. Juni 2011 das Bundeswahlgesetz zu novellieren, um das Auftreten negativer Stimmgewichte zu vermeiden. ${ }^{1}$ Bereits im Vorfeld der Bundestagswahl 2009 brachte die Fraktion Bündnis 90/Die Grünen einen diesbezüglichen Gesetzentwurf ein, der allerdings keine Mehrheit fand. ${ }^{2}$ Eine anhaltende öffentliche Diskussion wurde dadurch nicht angestoßen, doch hat die wissenschaftliche Literatur diverse Problemanalysen und Lösungsperspektiven in den Raum gestellt. ${ }^{3}$ Die im Folgenden skizzierten „systemnahen" Vorschläge wie auch alle anderen gehen sämtlich davon aus, dass der verfassungsgerichtliche Gesetzgebungsauftrag auf den vollständigen Ausschluss negativer Stimmgewichte gerichtet ist (II). Erlaubt der Urteilsspruch des Bundesverfassungsgerichts dagegen eine zwar theoretisch nicht ganz vollständige, aber dennoch praktisch weitestgehende Vermeidung negativer Stimmgewichte, bleibt dem Gesetzgeber ein größerer Spielraum, um das Wahlrecht zu modifizieren (III). Vor diesem Hintergrund erweitern wir die Diskussion über die notwendige Änderung des Wahlrechts um eine Handlungsoption, die wir als ,,schonende Verbindung" von Personen- und Verhältniswahl bezeichnen (IV) und die aus vielerlei Sicht attraktiv erscheint (V). Ihre Realisierbarkeit bringen wir in einem konkreten Gesetzgebungsvorschlag zum Ausdruck (VI).

1 BVerfGE 121 (2009) 266-317. - Negative Stimmgewichte wurden erstmals thematisiert von Hans Meyer, Der Überhang und anderes Unterhaltsames aus Anlaß der Bundestagswahl 1994, Kritische Vierteljahresschrift für Gesetzgebung und Rechtswissenschaft 77 (1994) 312-362 (321).

2 Der Antrag (Bundestagsdrucksache 16/11885) wurde am 3. Juli 2009 in namentlicher Abstimmung mit 97 Ja-Stimmen gegen 493 Nein-Stimmen bei 5 Enthaltungen abgelehnt.

3 Hans Herbert von Arnim, Verfassungswidrigkeit des Bundeswahlgesetzes aufgrund des "negativen Stimmgewichts" - Anmerkungen zum Urteil des Bundesverfassungsgerichts vom 3. Juli 2008, Recht und Politik 44 (2008) 136-138. Heiko Holste, Die Bundestagswahl und das verfassungswidrige Wahlgesetz, Recht und Politik 45 (2009) 152-156. Michael König, Paradoxie des negativen Stimmgewichts, Zeitschrift für Gesetzgebung 24 (2009) 177-195. Dieter Nohlen, Erfolgswertgleichheit als fixe Idee oder: Zurück zu Weimar? Zum Urteil des Bundesverfassungsgerichts über das Bundeswahlgesetz vom 3. Juli 2008, Zeitschrift für Parlamentsfragen 40 (2009) 179-195. Gerald Roth, Negatives Stimmgewicht und Legitimationsdefizite des Parlaments, Neue Zeitschrift für Verwaltungsrecht 27 (2008) 1199-1201. 


\section{Systemnahe Optionen}

Bisherige Vorschläge zum Ausschluss negativer Stimmgewichte setzen konsequenterweise an deren Ursachen an. ${ }^{4}$ Negative Stimmgewichte können entstehen, weil das geltende Wahlrecht eine Personenwahl- und eine Verhältniswahlkomponente vorsieht und zudem die föderale Untergliederung berücksichtigt. Je nachdem, welche Verbindung dazwischen hergestellt wird, ergeben sich unterschiedliche Handlungsoptionen.

Die hier resümierten vier Optionen bezeichnen wir als „systemnah", weil sie das bewährte Zweistimmensystem beibehalten. ${ }^{5}$ Option $\mathrm{F}$ orientiert sich an der föderalen Staatsgliederung und behandelt jedes Bundesland als ein separates Wahlgebiet (1). Erstund Zweitstimmen entwickeln dann einen völlig anderen Charakter mit entsprechenden verfassungsrechtlichen Problemen. Option G beschreibt das bereits vom Bundesverfassungsgericht angesprochene Grabenwahlsystem (2). Hier wird die Repräsentanz der Wähler kleinerer Parteien praktisch halbiert, was politisch und gesellschaftlich schwer vermittelbar sein dürfte.

Bei Option P dominiert die Personenwahlkomponente über die Verhältniswahlkomponente (3). Dagegen legt Option V das Schwergewicht auf die Verhältniswahlkomponente (4). Beide Optionen reproduzieren das geltende Wahlsystem in den Situationen, in denen es nicht zu Überhangmandaten kommt. Nur wenn im geltenden System Überhangmandate entstünden, wird korrigierend eingegriffen. Option P schützt das Personenwahlergebnis und korrigiert auf der Verhältniswahlseite, Option V geht umgekehrt vor.

Jenseits systemnaher Korrekturen gibt es mehr Freiheiten für eine Umgestaltung des Wahlsystems. Zum Beispiel favorisiert Behnke die Einführung von Zweipersonenwahlkreisen, Meyer ein Einstimmensystem mit Doppelauswertung als Wahlkreis- und Landeslistenstimme und Prittwitz ein Einstimmensystem mit impliziter Bundesliste. ${ }^{6}$

4 Hans Meyer, Lösungsmöglichkeiten nach dem Wahlrechtsurteil des BVerfG vom 3. Juli 2008, Deutsches Verwaltungsblatt 124 (2009) 137-146. Josef Isensee, Funktionsstörung im Wahlsystem: das negative Stimmgewicht - Denkbare Lösungen eines Dilemmas, Deutsches Verwaltungsblatt 125 (2010) 269-277.

5 Für mehr Einzelheiten zu den vier Optionen F, G, P und V siehe Friedrich Pukelsheim/Matthias Rossi, Wahlsystemnahe Optionen zur Vermeidung negativer Stimmgewichte, JuristenZeitung 65 (2010) 922-927.

6 Joachim Behnke, Überhangmandate und negatives Stimmgewicht: Zweimannwahlkreise und andere Lösungsvorschläge, Zeitschrift für Parlamentsfragen 41 (2010) 247-260. Hans Meyer, Die Zukunft des Bundestagswahlrechts zwischen Unverständnis, obiter dicta, Interessenkalkül und Verfassungsverstoß, Baden-Banden, 2010. Volker von Prittwitz, Anders wählen: Vom widersprüchlichen Als ob zur personalisierten Verhältniswahl, Manuskript. — Siehe auch Joachim Behnke, Die Bombe tickt weiter: Das immer noch existierende Problem der Überhangmandate und mögliche Lösungen, in: Wahlen und Wähler - Analysen aus Anlass der Bundestagswahl 2002. Hrsg. Oscar W. Gabriel/Bernhard Weßels/Jürgen W. Falter. Wiesbaden, 2005, 459-483 (470ff.), sowie den Sonderband 2009 "Wahlsystemreform" der Zeitschrift für Politikwissenschaft mit Beiträgen von Gerd Strohmeier, Dieter Nohlen, Florian Hartleb, Eckhard Jesse, Jürgen Falter, Florian Grotz, Hans Herbert von Arnim und Lasse Cronqvist/Uwe Jun. 


\section{Option F-Föderales Eigenständigkeitsmodell}

Eine Möglichkeit, den Anfall von Überhangmandaten und damit das Auftreten negativer Stimmgewichte auszuschließen, ist eine separat für jedes Bundesland vorgenommene Auswertung der Wahlergebnisse. Dazu müssten vorweg — etwa in der Mitte der Wahlperiode zusammen mit dem Bericht der Wahlkreiskommission — die Gesamtsitze des Bundestages auf die einzelnen Bundesländer verteilt werden. Am Wahltag selbst würde dann das zugewiesene Sitzkontingent im Verhältnis der Stimmenerfolge weiter verrechnet, einzeln für jedes der sechzehn Bundesländer.

Option F gerät in Konflikt mit dem unitarischen Charakter des Bundestages, dessen Abgeordnete nach Art. 38 Abs. 1 GG Vertreter des ganzen Volkes sind und nicht sechzehn separate Landesvölker repräsentieren. ${ }^{7}$ Als fatal dürfte sich erweisen, dass Zweitstimmen für Überhangsparteien erfolglos verpufften. ${ }^{8}$ So wären 2009 etwa ein Fünftel aller Zweitstimmen wirkungslos gewesen und nicht „maßgeblich", wie der Stimmzettelaufdruck verspricht. Den Wählern würde die Wahlgleichheit vorenthalten, die Art. 38 Abs. 1 GG gebietet.

\section{Option $G-$ Grabenwahlsystem}

Eine andere Möglichkeit bietet das Grabenwahlsystem, bei dem eine Hälfte des Bundestages durch eine reine Mehrheitswahl und die andere Hälfte durch eine reine Verhältniswahl bestimmt werden. Grundsätzlich könnten dafür die gesetzlichen Voraussetzungen wie gleich große Wahlkreise und Wahlkreis-Nachwahlregelungen geschaffen werden, die verbleibende Zeit bis zum 30. Juni 2011 reicht dafür wohl nicht mehr aus.

Option $\mathrm{G}$ beschränkt sich nicht auf eine Minimalkorrektur beanstandeter Ausnahmemängel, sondern geht mit einer radikalen Umgewichtung einher. Die Repräsentation der Wähler kleinerer Parteien wird um die Hälfte gekürzt und die so frei gemachten Sitze werden den größeren Parteien zugeleitet.

\section{Option P-Personenwahl über Verhältniswahl}

Dieser Vorschlag sieht die mit der Erststimme ausgeübte Personenwahl als sakrosankt an. Alle Wahlkreissieger erhalten wie bisher ein Mandat. An diese Vorgabe wird die Verhältnisrechnung angepasst. Auf der Ebene der Oberzuteilung, in der die Gesamtsitze des Bundestages den Parteien im Verhältnis ihrer bundesweiten Zweitstimmenerfolge zugeteilt werden, wird die Gesamtsitzzahl solange erhöht, bis alle Wahlkreissieger durch die Verhältnisrechnung getragen werden. Bei der Wahl 2009 hätte dies zu einer Bundestagsgröße von 641 Sitzen geführt, um den 45 CSU-Wahlkreissiegern einen Sitz zu-

7 Hans Meyer, Lösungsmöglichkeiten nach dem Wahlrechtsurteil des BVerfG vom 3. Juli 2008, Deutsches Verwaltungsblatt 124 (2009) 137-146 (140).

8 Joachim Behnke, Negatives Stimmgewicht, Erfolgswert und Überhangmandate - einige Anmerkungen, Kritische Vierteljahresschrift für Gesetzgebung und Rechtswissenschaft 93 (2010) $3-28(25)$. 
teilen zu können und dem jetzigen Überhang von drei CSU-Sitzen aus dem Weg zu gehen. ${ }^{9}$

Bei den Unterzuteilungen, in denen pro Partei die bundesweit zugeteilten Sitze an die Landeslisten weitergereicht werden, wendet Option P das direktmandatsbedingte Divisorverfahren mit Standardrundung (Augsburger Zuteilungsverfahren) an. Dieses Verfahren garantiert jeder Landesliste mindestens so viele Sitze, wie es im Land Wahlkreissieger gibt, und unterwirft nur die darüber hinaus gehenden Sitze der Verhältnisrechnung. Im Endergebnis werden Personen- und Verhältniswahl so verbunden, dass das bessere Ergebnis — die Direktmandate oder die verbleibenden Verhältnissitze zum Tragen kommen. ${ }^{10}$

Im Ergebnis erweist sich, dass Option P dem geltenden System absolut gleich kommt, solange im Anwendungsfall keine Überhangmandate auftreten. Nur in den Situationen, in denen Überhangmandate auftreten, weicht Option P vom geltenden System ab.

\section{Option $V-$ Verhältniswahl über Personenwahl}

Option V bricht mit dem Automatismus, jedem Wahlkreissieger ein Mandat zu garantieren. Zunächst werden in Ober- und Unterzuteilungen die Verhältnisrechnungen durchgeführt. Deren Ergebnisse bestimmen, wie viele Sitze für die Wahlkreissieger maximal zur Verfügung stehen.

Dabei kann es vorkommen, dass die Verhältnissitze nicht für alle Wahlkreissieger ausreichen und einige ohne Mandat bleiben. Es muss also abstrakt und ex ante eine Identifizierungsregel festgelegt werden, welche Wahlkreissieger ein Mandat erhalten und welche nicht. Option V benutzt dazu die genauen Erststimmenzahlen und schließt die Wahlkreissieger mit den wenigsten Erststimmen von der Sitzzuteilung aus. ${ }^{11}$ Dies erscheint freilich nur bei annähernd gleich großen Wahlkreisen gerecht.

Auch Option V reproduziert das geltende System im Regelfall, in dem keine Überhangmandate anfallen. Andernfalls bleiben so viele von den erststimmenschwächeren Wahlkreissiegern ohne Mandat, wie die Unterschiedszahl vorgibt. Die Parlamentsgröße von 598 Bundestagssitzen wird genau eingehalten. Option V wurde in Bayern für die Landtagswahl 1954 praktiziert und vom Bayerischen Verfassungsgerichtshof als ver-

9 Für dieses Vorgehen ist es unabdingbar, die Verteilungsrechnung mit dem Divisorverfahren mit Standardrundung (Sainte-Laguë/Schepers) durchzuführen, wie es das BWahlG seit 2008 vorschreibt. Das früher benutzte Hare-Quotenverfahren mit Ausgleich nach größten Resten (Hare/Niemeyer) taugt dafür nicht. Wird beim Quotenverfahren die Bundestagsgröße um einen Sitz erhöht, ist nicht garantiert, dass genau eine Landesliste einen Sitz mehr bekommt und alle anderen gleich bleiben. Es können auch zwei Landeslisten je einen Sitz mehr bekommen und dafür eine dritte Liste einen Sitz verlieren (Mandatszuwachs-Paradox, AlabamaParadox).

10 Friedrich Pukelsheim, Bundeswahlgesetz-Nächste Etappe, Deutsches Verwaltungsblatt 123 (2008) 889-897 (894).

11 Alternativ könnte man den Ausschluss an den prozentualen Erststimmenanteilen orientieren. 
fassungsgemäß beurteilt. Logik und Argumentation des Urteils dürften wohl auch im Licht der heutigen Rechtsprechung Bestand haben. ${ }^{12}$

\section{Inhalt und Bedeutung des Gesetzgebungsauftrags}

Die von uns zusätzlich zu diesen Optionen sogleich vorgeschlagene Möglichkeit, die Personenwahl mit der Verhältniswahl auf eine schonende Weise (Option S) zu verbinden, reduziert die Anzahl von Überhangmandaten und mindert damit zugleich die Möglichkeit negativer Stimmgewichte, schließt sie aber nicht vollständig aus. Vor diesem Hintergrund fragt es sich, ob die gesetzliche Einführung der schonenden Verbindung überhaupt mit dem Auftrag vereinbar ist, den das Bundesverfassungsgericht dem Gesetzgeber in seinem Urteil vom 3. Juli 2008 auferlegt hat. Zur Beantwortung dieser Frage ist zunächst in gebotener Kürze die allgemeine Bedeutung verfassungsgerichtlicher Gesetzgebungsaufträge zu beleuchten (1), bevor sodann der konkrete Inhalt und Umfang des Gesetzgebungsauftrags vom 3. Juli 2008 untersucht wird (2).

\section{Verfassungsgerichtliche Gesetzgebungsaufträge}

Verfassungsgerichtliche Gesetzgebungsaufträge berühren in besonders deutlicher Weise das Spannungsverhältnis zwischen dem demokratischen und dem rechtsstaatlichen Prinzip, die beide gemäß Art. 20 und Art. 28 Abs. 1 GG die verfassungsrechtliche Ordnung im Bund und in den Ländern prägen. Während das Demokratieprinzip für eine weitgehende Gestaltungsfreiheit des parlamentarischen Gesetzgebers steht, verlangt das Rechtstaatssprinzip die Beachtung der rechtlichen Grenzen, die in Gestalt der verfassungsmäßigen Ordnung gemäß Art. 20 Abs. 3 Hs. 1 GG sowie gemäß Art. 1 Abs. 3 GG insbesondere der Grundrechte auch für den Gesetzgeber gelten. Das Bundesverfassungsgericht ist nach der Gewaltenteilung des Grundgesetzes die einzige Instanz, die die in Recht gegossenen politischen Entscheidungen des unmittelbar demokratisch legitimierten Gesetzgebers bei Überschreitung dieser Grenzen verwerfen kann.

Zwischen dieser Befugnis, bereits beschlossene Bestimmungen zu verwerfen, und dem Auftrag, binnen einer bestimmten Zeit neue Regelungen zu erlassen, besteht gleichwohl ein gradueller Unterschied, wird doch bei der Verwerfung nur die das „Wie" betreffende Gestaltungsfreiheit, bei dem Auftrag dagegen schon die das „Ob" betreffende Entschließungsfreiheit des Gesetzgebers berührt. Zu einer offenen Rebellion des parlamentarischen Gesetzgebers, der sich mit Gesetzgebungsaufträgen des Bundesverfassungsgerichts konfrontiert sieht, ist es in der Vergangenheit gleichwohl aus guten Gründen nie gekommen. Zum einen ist die Entschließungsfreiheit des parlamentarischen Gesetzgebers im modernen und offenen Verfassungsstaat ohnehin nicht mehr unbeschränkt. Internationale und vor allem supranationale Vorgaben nehmen den Gesetzgeber ebenso in die Pflicht wie die Verfassung selbst, ${ }^{13}$ die an verschiedenen Stellen

12 BayVerfGH 7 (1954) 99-106. Siehe auch Klaus Unterpaul, Die Grundsätze des Landeswahlrechts nach der Bayerischen Verfassung im Lichte der Entwicklung von 1946 bis 1989, München, 1992 (80-83, 176-179).

13 Vgl. zum Ganzen Thilo Brandner, Gesetzesänderung, 2004, S. 169 ff. 
— und so auch in Art. 38 Abs. 3 GG - nach gesetzlicher Ausgestaltung der verfassungsrechtlich nur rudimentär und grundsätzlich verankerten Vorgaben verlangt.

Zum anderen greift das Bundesverfassungsgericht regelmäßig nur dort zum Instrument eines konkreten Gesetzgebungsauftrags, wo die beanstandete Regelung so bedeutsam ist, dass ihre sofortige Außerkraftsetzung zu noch verfassungswidrigeren Folgen führen würde als ihre Beibehaltung. Gesetzgebungsaufträge stellen sich vor diesem Hintergrund nicht als schärfere, sondern als mildere Konsequenz eines Verfassungsverstoßes dar: Die beanstandete Regelung wird nicht sofort unanwendbar, sondern bleibt in Kraft, bis der Gesetzgeber eine verfassungskonforme Regelung erlassen hat. Insofern beschneidet das Bundesverfassungsgericht die Entschließungsfreiheit des Gesetzgebers gerade zur Wahrung dessen Gestaltungsfreiheit. Verfassungsgerichtliche Gesetzgebungsaufträge sind deshalb — vorbehaltlich ihres konkreten Inhalts — grundsätzlich nicht nur zulässig, sondern besonderer Ausdruck der Gewaltenteilung zwischen dem verfassungswahrenden Bundesverfassungsgericht und dem politisch agierenden Gesetzgeber. Sie können letztlich als Ausdruck einer aufschiebend befristeten Verfassungswidrigkeit verstanden werden, weil die beanstandete Regelung nicht sofort, sondern erst mit Verstreichen des Fristablaufs für den Gesetzgebungsauftrag nichtig wird.

In Bezug auf den Gesetzgebungsauftrag vom 3. Juli 2008 stellt sich der verfassungsgerichtliche Eingriff in die Entschließungsfreiheit des Gesetzgebers zudem deshalb noch milder dar, weil er an sich nur den verfassungsrechtlichen Gesetzgebungsauftrag des Art. 38 Abs. 3 GG wiederholt und präzisiert. Dem Gesetzgeber eine Frist für den Erlass einer verfassungsgemäßen Regelung zu setzen, steht im Einklang mit der verfassungsrechtlichen Gewaltenteilung und ist Ausdruck der verfassungsrechtlichen Verantwortung des Bundesverfassungsgerichts, nicht etwa Ausdruck einer kompetenzüberschreitenden Anmaßung von politischen Gestaltungsbefugnissen.

\section{Inhalt und Umfang des Gesetzgebungsauftrags}

Schwieriger ist die Frage nach Inhalt und Umfang des konkreten Gesetzgebungsauftrags zu beantworten. Das Bundesverfassungsgericht formuliert im Leitsatz 2 seines Urteils die Verpflichtung des Gesetzgebers, spätestens bis zum 30. Juni 2011 eine verfassungsgemäße Regelung zu treffen. Dieser für sich genommen unbestimmte Auftrag bezieht sich auf die im Leitsatz 1 formulierte Feststellung, dass $\S 7$ Abs. 3 Satz 2 i.V.m. $\S 6$ Abs. 4 und 5 BWahlG die Grundsätze der Gleichheit und Unmittelbarkeit verletzt, ,,soweit hierdurch ermöglicht wird, dass ein Zuwachs an Zweitstimmen zu einem Verlust an Sitzen der Landeslisten oder ein Verlust an Zweitstimmen zu einem Zuwachs an Sitzen der Landeslisten führen kann". Dass dem Wortlaut dieser Formulierung nach schon die bloße Möglichkeit des Auftretens negativer Stimmgewichte die Verletzung der genannten Wahlrechtsgrundsätze auslösen soll und deshalb verfassungswidrig ist, indiziert zunächst, dass die neu zu schaffende Regelung schon jede theoretische Möglichkeit dieser Effekte ausschließen soll, und sei sie noch so unwahrscheinlich. Bei näherer Betrachtung haben wir allerdings aus mindestens vier Gründen Zweifel, dass die Feststellungswirkung des Bundesverfassungsgerichts und mit ihr das Normwiederholungsverbot so kompromisslos zu verstehen sind. 
Erstens ist der gesetzgeberische Gestaltungsraum bei der notwendigen Konkretisierung des Wahlrechts nicht nur durch einen Fluchtpunkt determiniert, sondern durch vielerlei Vorgaben begrenzt. Die die Wahlentscheidung und -wirkung des Wählers schützenden Wahlrechtsgrundsätze des Art. 38 Abs. 1 Satz 1 GG sind ebenso zu beachten wie die Chancengleichheit der Parteien aus Art. 21 Abs. 1 GG i.V.m. Art. 3 Abs. 1 GG und die Statusrechte der Abgeordneten aus Art. 38 Abs. 1 Satz 1 GG. Hinzu kommt eine angemessene föderale Gewichtung, wie sie zwar nicht unmittelbar von Art. 38 GG vorgegeben wird, jedoch aus dem bundesstaatlichen Charakter der grundgesetzlichen Staatsordnung folgt. Das gesamte Wahlsystem ausschließlich an dem Ausschluss auch nur der Möglichkeit negativer Stimmgewichte auszurichten, engte diesen ohnehin begrenzten Gestaltungsspielraum des Gesetzgebers über Gebühr ein und nähme ihm vor allem die Möglichkeit, andere Gesichtspunkte in den Vordergrund zu stellen.

Auch die konkreten Ausführungen des Bundesverfassungsgerichts sprechen zweitens gegen ein striktes Verbot negativer Stimmgewichte. Während der Gesetzgebungsauftrag in seiner in den Leitsätzen 1 und 2 zusammengefassten Kürze in der Tat recht absolut klingt, gibt seine Begründung im Urteil selbst zu erkennen, dass das Bundesverfassungsgericht um die Schwierigkeiten der vielfältigen Anforderungen an das Wahlsystem weiß. Hier ist die Rede von einem „komplexen Regelungssystem" und es wird anerkannt, dass ,,selbst eine geringfügige Änderung des Bundeswahlgesetzes [...] in der vorliegenden Konstellation möglicherweise zu weit reichenden strukturellen Veränderungen [führt]". ${ }^{14}$ Das Bundesverfassungsgericht schlussfolgert aus dieser Komplexität zwar expressis verbis nur die Länge der Frist, die es dem Gesetzgeber für die Neugestaltung des Wahlrechts einräumt. Zugleich nimmt es aber bewusst in Kauf, dass es bei den zwischenzeitlich erfolgten Wahlen im Herbst 2009 zu negativen Stimmgewichten kommt. Negative Stimmgewichte sind somit nicht um jeden Preis zu vermeiden, sondern sind der Abwägung mit anderen Belangen zugänglich.

Drittens hat das Bundesverfassungsgericht in seinen früheren Entscheidungen zum Wahlrecht diese fast schon als magisch zu bezeichnende, weil nicht gleichmäßig und vollständig einzuhaltende verfassungsrechtliche Begrenzung des gesetzgeberischen Gestaltungsspielraums regelmäßig anerkannt. Das negative Stimmgewicht wurde bereits — wenn auch unter anderen Bezeichnung — im Rahmen der Klage Niedersachsens anlässlich der Bundestagswahl von 1994 im Verfahren vor dem Bundesverfassungsgericht thematisiert, ${ }^{15}$ ohne dass sich das Bundesverfassungsgericht näher mit ihm befasst geschweige es denn für verfassungswidrig erklärt hätte. Viele Kommentatoren in der Wissenschaft haben deshalb auf den Widerspruch zwischen der jetzigen Feststellung der Verfassungswidrigkeit und der Begründung der Entscheidung zu den Überhang-

14 BVerfGE 121 (2009) 266-317 (314 f.).

15 Hans Meyer, Der Überhang und anderes Unterhaltsames aus Anlaß der Bundestagswahl 1994, Kritische Vierteljahresschrift für Gesetzgebung und Rechtswissenschaft 77 (1994) 312-362. Dies betonend auch Joachim Behnke, Negatives Stimmgewicht, Erfolgswert und Überhangmandate - einige Anmerkungen, Kritische Vierteljahresschrift für Gesetzgebung und Rechtswissenschaft 93 (2010) 3-28 (7). 
mandaten hingewiesen. ${ }^{16}$ Nun mag es selbstverständlich sein, dass das Bundesverfassungsgericht - nicht zuletzt auch wegen wechselnder personeller Besetzung — seine eigene Rechtsprechung - zumeist behutsam - korrigiert. Ebenso mag es sein, dass das Bundesverfassungsgericht in seinem Urteil vom 3. Juli 2008 weniger auf die negativen Stimmgewichte als vielmehr auf die Überhangmandate zielte. Doch entscheidend scheint bei einer Berücksichtigung der konkreten Umstände dieses Urteils vielmehr zu sein, dass die negativen Stimmgewichte in der Konstellation der Nachwahl in einem Wahlkreis ,taktisch" genutzt werden konnten und dass den Wählern die Wahlurne nicht als eine black box erschien, sondern jedenfalls teilweise transparent. Vor diesem Hintergrund zielt die so kompromisslos klingende Unzulässigkeit negativer Stimmgewichte eher als ein Versuch, ein taktisches Wählen zu unterbinden. Unabhängig von der Frage, wie ein solches taktisches Wählen von einer ,echten" oder „wahren" Wahlentscheidung unterschieden werden kann, ist insofern aber hervorzuheben, dass ein solches Wahlverhalten eben nur in der besonderen Konstellation der Nachwahl möglich ist. Wenn keine Nachwahlen stattfinden, können negative Stimmgewichte nicht operationalisiert werden.

Eine solche Relativierung des Verbots negativer Stimmgewichte erscheint uns viertens vor allem deshalb geboten, weil das Wahlrecht insgesamt nicht von der Umsetzung abstrakter Ideale, sondern von seinem konkreten Gebrauch geprägt sein soll. Mehr als in jedem anderen Bereich fordert das Bundesverfassungsgericht den Gesetzgeber immer wieder dazu auf, das Wahlrecht als law in action zu beobachten und das law in the books gegebenenfalls entsprechend anzupassen. ${ }^{17}$ Vor diesem Hintergrund kann es dem Bundesverfassungsgericht nicht darum gehen, bereits die Möglichkeit negativer Stimmgewichte im Interesse der Reinheit des Wahlsystems auszuschließen, sondern in erster Linie darum, diesen Effekt nicht im konkreten Wahlverhalten ausnutzbar zu gestalten. Eine Regelung, die durch die Reduzierung der Überhangmandate auch die Wahrscheinlichkeit negativer Stimmgewichte verringert und zudem durch geeignete Nachrückerregelung die Notwendigkeit von Nachwahlen vermeidet, genügt dem Gesetzgebungsauftrag des Bundesverfassungsgerichts daher in jeder Beziehung.

Nachwahlen nämlich führen immer zu einer massiven Beeinträchtigung des Grundsatzes der Wahlgleichheit. Es ist deshalb erstrebenswert, Nachwahlen wenn irgend möglich zu vermeiden, und dieses Bestreben hat nur am Rande mit negativen Stimmgewichten zu tun. Da die schonende Verbindung das Verhältnis von Personenwahl und Verhältniswahl neu regelt und die beiden Komponenten auch rechnerisch aufeinander

16 Dieter Nohlen, Erfolgswertgleichheit als fixe Idee oder: Zurück zu Weimar? Zum Urteil des Bundesverfassungsgerichts über das Bundeswahlgesetz vom 3. Juli 2008, Zeitschrift für Parlamentsfragen 40 (2009) 179-195. Gerald Roth, Negatives Stimmgewicht und Legitimationsdefizite des Parlaments, Neue Zeitschrift für Verwaltungsrecht 27 (2008) 1199-1201. Hans Meyer, Lösungsmöglichkeiten nach dem Wahlrechtsurteil des BVerfG vom 3. Juli 2008, Deutsches Verwaltungsblatt 124 (2009) 137-146. Heinrich Lang, Stellungnahme zur öffentlichen Anhörung des Innenausschusses des Deutschen Bundestages am 4. Mai 2009 in Berlin.

17 BVerfGE 95 (1997) 408-425 (418 f.): "Der Gesetzgeber muss sich bei seiner Einschätzung und Bewertung nicht an abstrakt konstruierten Fallgestaltungen, sondern an der politischen Wirklichkeit orientieren." Vergleiche dazu Markus Heintzen, Die Bundestagswahl als Integrationsvorgang - Besprechung des Grundmandatsurteils des Bundesverfassungsgerichts, Deutsches Verwaltungsblatt 112 (1997) 744-749. 
verweist, sehen wir es als eine willkommene zusätzliche Möglichkeit an, diese Bindung auch zur Vermeidung von Nachwahlen einzusetzen.

In diesen Überlegungen sehen wir eine hinreichende Rechtfertigung, im folgenden Abschnitt IV eine schonende Verbindung zwischen Personen- und Verhältniswahl (Option S) zu entwickeln und ihre Güteeigenschaften zu diskutieren (V). Sollte die verfassungsrechtliche Vorgabe doch verlangen, auch die Restwahrscheinlichkeiten eines Auftretens negativer Stimmgewichte zum Verschwinden zu bringen, oder sollte es sich nach ein- oder zweimaliger Anwendung erweisen, dass trotz schonender Verbindung der Anfall von Überhangmandaten neuer Art nicht im erhofftem Maße rückläufig ist, so bietet sich als verschärfte Variante Option W an (V.7), in der negative Stimmgewichte gänzlich ausgeschlossen sind, deren systemstabilisierenden Selbstheilungskräfte allerdings hinter denen von Option S zurückbleiben.

\section{Option S-Schonende Verbindung}

Unser Vorschlag basiert auf zwei Ausgangsüberlegungen. Erstens sollte die Umsetzung des verfassungsgerichtlichen Nachbesserungsauftrags solange möglichst systemnah erfolgen, wie es weder von den politischen Akteuren noch in der Öffentlichkeit Hinweise für eine grundsätzliche Umgestaltung des Wahlsystems gibt. In diesem Sinn zielt Option $\mathrm{S}$ auf eine Neuregelung nur solcher Situationen, die unter dem geltenden Recht Probleme bereiten.

Zweitens orientieren wird uns an dem in $\S 1$ I BWahlG formulierten Ziel, die Abgeordneten des Deutschen Bundestages nach den Grundsätzen einer mit der Personenwahl verbundenen Verhältniswahl zu wählen. Wir konzentrieren uns auf das verbindende Element zwischen der Personenwahl und der Verhältniswahl, wobei die Einbeziehung der föderalen Struktur unangetastet bleibt. Diese Verbindung steht für uns als ein eigenständiger und ebenso gestaltungsfähiger wie gestaltungsbedürftiger Baustein im Mittelpunkt. ${ }^{18}$ Die schonende Verbindung nimmt somit eine vermittelnde Position zwischen den Optionen P und V ein, in denen kompromisslos jeweils eine Systemkomponente die andere dominiert.

Ein Fixpunkt unseres Vorschlags ist das auch im geltenden Wahlrecht vorhandene Bestreben, jeden Wahlkreis durch einen Abgeordneten im Bundestag zu vertreten. Anders als das geltende Wahlrecht gehen wir aber nicht davon aus, dass ausschließlich der Wahlkreissieger den Wahlkreis repräsentieren kann. Als Wahlkreissieger bezeichnen wir denjenigen Bewerber im Wahlkreis, der die meisten Erststimmen erhält. In den allermeisten Fällen wird der Wahlkreissieger ein Mandat erhalten.

Jedoch wird der Automatismus der Mandatsvergabe an Wahlkreissieger zu Gunsten der Minderung negativer Stimmgewichte aufgeben. Einen Wahlkreis, der von diesem Automatismus ausgenommen ist, bezeichnen wir als Ausnahmewahlkreis. In Ausnah-

18 Dagegen geht bei der Charakterisierung des Systems als "personalisierte Verhältniswahl" erst sprachlich und dann auch gedanklich die Bedeutung verloren, die der Verbindung zukommt. Aus unserer Sicht ist es die mangelhaft ausgestaltete Verbindung, die den momentanen Schwierigkeiten zu Grunde liegt. Akzeptiert man die herzustellende Verbindung als einen eigenständigen Gestaltungsfaktor, eröffnen sich neue Wege, um dem Entstehen von Überhangmandaten und dem Auftreten negativer Stimmgewichte entgegen zu wirken. 
mefällen kann die Parlamentsvertretung von Listenkandidaten anderer Parteien wahrgenommen werden, die zwar nach Erststimmen unterlegen sind, aber mittels Zweitstimmen über eine Landesliste gewählt wurden. Entscheidend ist, dass der Wahlkreis überhaupt im Bundestag vertreten ist. ${ }^{19}$

Der Verzicht auf die parlamentarische Vertretung von Wahlkreisen zwingend und ausschließlich nur durch Wahlkreissieger ist zwar bei juristischer, nicht aber bei praktischer Betrachtung ein gewisser Paradigmenwechsel. Denn praktisch trifft es nur auf etwa ein Drittel der Mitglieder des Bundestages zu, dass sie alleine als Wahlkreissieger aus ihrem Wahlkreis kommen und nicht auch weitere Abgeordnete. Dies belegt, dass bei einer mit der Personenwahl verbundenen Verhältniswahl der Verweis auf „,meinen Wahlkreis" bei weitem nicht dasselbe bedeutet wie bei einer reinen Mehrheitswahl. Der Verweis besagt auch im besten Fall nicht mehr, als dass der Abgeordnete in besagtem Wahlkreis zu Hause ist und hier den Schwerpunkt seiner politischen Arbeit außerhalb des Parlaments sieht. Oft wird aber sogar dieses Kriterium nicht gegeben sein, denn so manche Bewerber wohnen gar nicht in ,ihrem" Wahlkreis, sondern „bewerben sich in einem Wahlkreis", um ihren Einzug in den Bundestag bei hinteren Listenplätzen zusätzlich abzusichern.

Der Ausnahmefall, in dem der Wahlkreis nicht durch den Wahlkreissieger, sondern durch einen oder mehrere Listenkandidaten anderer Parteien vertreten wird, tritt nur ein, wenn es mehr Wahlkreissieger als Verhältnissitze gibt. ${ }^{20}$ Nach geltendem Recht werden dann „Überhangmandate" ins Leben gerufen, die vom BWahlG als Unterschiedszahl benannt werden. ${ }^{21}$ Angesichts der Vor-, Rück- und Querverweise in $\S \S 6-7$ BWahlG kann die Unterschiedszahl zum einen in der Oberzuteilung auftreten, in der die 598 Bundestagssitze den Parteien im Verhältnis ihrer Zweitstimmen zugeteilt werden. Andererseits kann eine solche Unterschiedszahl in jeder Unterzuteilung zum Vorschein kommen, in der die einer Partei insgesamt zustehenden Sitze ihren Landeslisten zugeteilt werden.

Die verwirrenden Verweisungen des geltenden Gesetzes verhindern eine normenklare und verständliche Beschreibung der Regelungen. Sie sind auch nicht sachgerecht, da sie Ungleiches gleichsetzen. Die Oberzuteilung regelt die parteipolitische Zusammensetzung des Bundestages. Im Gegensatz dazu orientieren sich die Unterzuteilungen an der föderalen Staatsgliederung, um die personale Zusammensetzung des Bundestages mitzubestimmen. Parteipolitische Repräsentation und föderale Repräsentation sind zwei verschiedene Anliegen.

Aus diesem Grund schlagen wir vor, die Oberzuteilung in der anvisierten schonenden Verbindung anders zu regeln als die Unterzuteilungen. In der Oberzuteilung verhindert eine maßvolle Erhöhung der Gesamtsitze des Bundestages, dass es zu Überhangman-

19 Zur Statusgleichheit der Abgeordneten statt vieler Martin Morlok, in: Horst Dreier (Herausgeber), GG-Kommentar, Band 2, 2. Auflage, 2006, Art. 38, Rn. 134.

20 Als "seltene Eventualität" schon diskutiert von Siegfried Geyerhahn, Das Problem der verhältnismäßigen Vertretung - Ein Versuch seiner Lösung. Wiener Staatswissenschaftliche Studien, Band 3, Heft 4, Herausgeber Edmund Bernatzik/Eugen von Philippovich, Tübingen 1902, S. 29. - Jedoch ist es seit 1980 bei Bundestagswahlen nicht der Regelfall, der regelmäßig eintritt, sondern die seltene Eventualität.

21 Der Begriff „Überhangmandat" kommt im Gesetz nicht vor. § 6 V BWahlG spricht nur davon, dass sich die Gesamtzahl der Sitze um eine „Unterschiedszahl” erhöht. 
daten kommt (1). In den Unterzuteilungen können Überhangmandate neuer Art anfallen, wenn auch deutlich weniger als nach alter Art (2). Wir illustrieren die schonende Verbindung jeweils anhand der Ergebnisse der letzten Bundestagswahl vom September 2009. ${ }^{22}$

\section{Schonende Oberzuteilung}

Entsprechend dem Streben nach einer systemnahen Modifizierung des geltenden Wahlrechts wirkt sich die schonende Oberzuteilung nicht im Regelfall, sondern nur im Ausnahmefall aus. In der bundesweiten Oberzuteilung aller Sitze an die Parteien geht sie wie folgt vor. In einer Anfangsrechnung werden die in $\S 1$ I BWahlG genannten 598 Gesamtsitze den Parteien im Verhältnis ihrer Zweitstimmen zugeteilt.

\section{a) Regelfall}

Der Regelfall tritt ein, wenn auf jede Partei mindestens so viele Verhältnissitze entfallen, wie sie bundesweit Wahlkreissieger vorweist. In diesem Fall ist die Oberzuteilung mit der Anfangsrechnung sofort beendet.

\section{b) Ausnahmefall}

Dagegen tritt der Ausnahmefall ein, wenn die Zahl der Wahlkreissieger die Zahl der Verhältnissitze übersteigt. Ein Ausnahmefall ist zum ersten und einzigen Mal bei der Wahl 2009 zu Stande gekommen, als die CSU 45 Wahlkreissieger stellte, aber nur 42 Verhältnissitze zugeteilt bekam. Davor waren Ausnahmefälle auf Oberzuteilungsebene unbekannt und mussten also auch nicht thematisiert werden.

Option S geht im Ausnahmefall so vor, dass sie die Wahlkreissieger nach fallenden Erststimmen reiht. ${ }^{23}$ Von den erststimmenstärkeren Wahlkreissiegern erhalten (nur) so viele ein Mandat, wie die Zahl der Verhältnissitze vorgibt. Auch im Ausnahmefall bleibt also für die 598 Ausgangssitze, die durch die Verhältnisrechnung abgedeckt sind, alles beim Alten.

Für die wenigen Ausnahmewahlkreise, in denen das nicht möglich ist, trifft Option S besondere Vorkehrungen. In diesen Ausnahmewahlkreisen wird geprüft, ob einer der Mitbewerber des Wahlkreissiegers (gemäß den weiteren Regelungen für die Unterzuteilungen) über seine Landesliste in den Bundestag einzieht und der Wahlkreis somit repräsentiert ist. Gibt es erfolgreiche Mitbewerber anderer Parteien, hat der Wahlkreis-

22 Für Vorhersagen zum Anfall von Überhangmandaten alter Art im Vorfeld der Wahl siehe Joachim Behnke, Überhangmandate bei der Bundestagswahl 2009 - Eine Schätzung mit Simulationen, Zeitschrift für Parlamentsfragen 40 (2009) 620-636.

23 Die Reihung nach Erststimmen und die Prüfung in den Ausnahmewahlkreisen, ob Mitbewerber über ihre Landesliste ein Mandat erhalten, ist mit einem beträchtlichen Aufwand verbunden. Hinsichtlich einer etwaigen Wahlprüfung ist zu bedenken, dass eine fehlerhafte Ermittlung der Erststimmen sich nicht mehr nur auf einen Wahlkreis auswirkt, sondern über den Wahlkreis hinaus auf das Gesamtergebnis ausstrahlen kann. 
sieger das Nachsehen und erhält kein Mandat. Falls alle Ausnahmewahlkreise durch Mitbewerber vertreten sind, findet die Oberzuteilung mit der Anfangsrechnung erneut ihr schnelles Ende.

Sofern es aber einen Ausnahmewahlkreis gibt, in dem kein Mitbewerber des Wahlkreissiegers in den Bundestag einrückt, wird das Anliegen der örtlichen Repräsentanz eines jeden Wahlkreises weiterverfolgt. Hier ist dann die Anfangsrechnung fortzusetzen, indem die Gesamtzahl der Bundestagssitze in Einerschritten wächst und die Verhältnissitze neu zugeteilt werden. Die Erhöhung findet ihr Ende, sobald alle Ausnahmewahlkreise durch Mitbewerber vertreten sind oder es keinen Ausnahmewahlkreis mehr gibt.

Zusammenfassend garantiert die schonende Verbindung von Personen- und Verhältniswahl in der Oberzuteilung, dass für alle Parteien ihre Sitze insgesamt im Verhältnis der Zweitstimmen zugeteilt sind. Gleichzeitig ist jeder Wahlkreis im Bundestag vertreten, allermeist durch Wahlkreissieger und nur ausnahmsweise durch Mitbewerber. In jedem Fall ist aus allen 299 Wahlkreisen mindestens einer der Bewerber aus den Kreiswahlvorschlägen in den Bundestag gewählt. Somit ist den Vorschriften des § 1 II BWahlG Genüge getan.

\section{c) Illustration am Beispiel der Wahl 2009}

Diese abstrakten Überlegungen seien anhand der konkreten Daten der Bundestagswahl 2009 hypothetisch illustriert, der sprachlichen Einfachheit halber im Indikativ. Die CDU hatte bundesweit 173 Wahlkreissieger vorzuweisen. Von den 598 Ausgangssitzen entfallen in der Anfangsrechnung 173 Verhältnissitze auf die CDU. Also werden alle CDUWahlkreissieger von den CDU-Verhältnissitzen getragen. Auch die anderen Parteien unterliegen dem Regelfall, dass alle Wahlkreissieger durch die Verhältnissitze gedeckt sind, bis auf die CSU. Die CSU sorgt für einen Ausnahmefall. Sie stellt in allen bayerischen Wahlkreisen den Wahlkreissieger (45). Von den 598 Ausgangssitzen teilt die Anfangsrechnung der CSU aber weniger Verhältnissitze zu (42), siehe Tabelle 1.

\begin{tabular}{|lc|cc|}
\hline \multicolumn{4}{|c|}{$\begin{array}{c}\text { Tabelle 1: Schonende Verbindung 2009 für 598 Sitze, } \\
\text { Anfangsrechnung für die Oberzuteilung }\end{array}$} \\
\hline & Zweitstimmen & $\frac{\text { Zweitstimmen }}{68196}$ & Verhältnissitze \\
\hline CDU & 11828277 & 173.4 & 173 \\
SPD & 9990488 & 146.497 & 146 \\
FDP & 6316080 & 92.6 & 93 \\
LINKE & 5155933 & 75.6 & 76 \\
GRÜNE & 4643272 & 68.1 & 68 \\
CSU & 2830238 & 41.501 & 42 \\
\hline Summe & 40764288 & & 598 \\
\multicolumn{4}{|c|}{ Aufje 68 196 Zweitstimmen entfällt rund ein Verhältnissitz. } \\
\hline
\end{tabular}


Somit tritt eine Unterschiedszahl ins Leben, nämlich $45-42=3$. Um die schonende Verbindung zu vollziehen, müssen die CSU-Wahlkreissieger nach fallenden Erststimmen gereiht werden. Die 42 erststimmenstärkeren Wahlkreissieger erhalten jeweils ein Mandat. Die letzten drei Rangplätze 43 bis 45 werden näher untersucht, siehe Tabelle 2 .

\begin{tabular}{|c|c|c|c|}
\hline \multicolumn{4}{|c|}{$\begin{array}{l}\text { Tabelle 2: Schonende Verbindung } 2009 \text { für } 598 \text { Sitze, } \\
\text { drei CSU-Ausnahmewahlkreise mit Siegerrängen 43-45 }\end{array}$} \\
\hline $\begin{array}{l}\text { Sieger- } \\
\text { rang }\end{array}$ & $\begin{array}{c}\text { Erst- } \\
\text { stimmen }\end{array}$ & $\begin{array}{c}\text { Partei(en) erfolgreicher } \\
\text { Mitbewerber }\end{array}$ & $\begin{array}{l}\text { Wahlkreis-Num } \\
\text { und Beschreib }\end{array}$ \\
\hline 43 & $9398 \quad 53$ & - & $227 \mathrm{D}$ \\
\hline 44 & $48943 \quad 37$ & $\mathrm{D}$, Lin & 244 Nü \\
\hline 45 & 47519 & SPD & 245 Nürnberg-Süd \\
\hline
\end{tabular}

Auf Rang 43 steht mit 49398 Erststimmen (53 Prozent der Erststimmen) der Sieger des Wahlkreises 227 (Deggendorf), auf Rang 44 mit 48943 Erststimmen (37 \%) der Sieger des Wahlkreises 244 (Nürnberg-Nord) und auf Rang 45 mit 47519 Erstimmen (39\%) der Sieger des Wahlkreises 245 (Nürnberg-Süd). Es zeigt sich, dass die beiden letztgenannten Wahlkreise bereits durch Abgeordnete anderer Parteien vertreten sind, die als Listenkandidaten einen Sitz errungen haben. Die dortigen Wahlkreissieger bekommen deshalb kein Mandat.

Im Wahlkreis 227 (Deggendorf) ist dagegen kein Mitbewerber über seine Landesliste gewählt. Um diesen Ausnahmewahlkreis nicht unvertreten zu lassen, wird die Gesamtzahl der Sitze in Einerschritten über die 598 Ausgangssitze hinaus erhöht, bis auch aus diesem Wahlkreis ein Abgeordneter gewählt wird. Bei 599 Abgeordneten gibt die Oberzuteilung ein zusätzliches Mandat an die SPD, das bei der anschließenden Unterzuteilung an ihre Landesliste in Bayern fällt. Auf dem zusätzlichen 17. Listenplatz rückt ein Kandidat ein, der sich im Wahlkreis 218 (München-Nord) beworben hat. Der Ausnahmewahlkreis 227 bleibt unberührt.

Der 600. Bundestagssitz geht an die CDU und somit nicht nach Bayern. Den 601. Sitz bekommen die Grünen zugeteilt. Dieser neue 11. Sitz entfällt auf eine Kandidatin in Bayern, die sich im Wahlkreis 216 (Fürstenfeldbruck) beworben hat. Wieder ist der Ausnahmewahlkreis 227 nicht betroffen. Die Sitze 602 bis 608 entfallen auf Landeslisten außerhalb Bayerns.

Bei der Gesamtzahl von 609 Sitzen erhöht sich die Anzahl der Verhältnissitze der FDP, wobei das FDP-Kontingent in Bayern von 14 auf 15 steigt. Auf dem 15. Listenplatz zieht der FDP-Bewerber des Wahlkreises 227 (Deggendorf) in den Bundestag ein. Jetzt ist auch der dritte Ausnahmewahlkreis durch einen erfolgreichen Mitbewerber vertreten, der Wahlkreissieger hat das Nachsehen. Somit ist mit 609 Gesamtsitzen das Ende der Erhöhung erreicht. Die endgültige Oberzuteilung ist in Tabelle 3 ausgewiesen. 
Tabelle 3: Schonende Verbindung 2009 für 609 Sitze, Endrechnung für die Oberzuteilung

\begin{tabular}{|c|c|c|c|c|}
\hline \multicolumn{2}{|c|}{ Zweitstimmen } & $\frac{\text { Zweitstimmen }}{66830}$ & $\begin{array}{l}\text { Verhältnis- } \\
\text { sitze }\end{array}$ & $\begin{array}{l}\text { Wahlkreis- } \\
\text { sieger }\end{array}$ \\
\hline $\mathrm{CDU}$ & 11828277 & 177.0 & 177 & 173 \\
\hline SPD & 9990488 & 149.49 & 149 & 64 \\
\hline FDP & 6316080 & 94.51 & 95 & 0 \\
\hline LINKE & 5155933 & 77.1 & 77 & 16 \\
\hline GRÜNE & 4643272 & 69.48 & 69 & 1 \\
\hline CSU & 2830238 & 42.3 & 42 & 42 \\
\hline Summe & 40764288 & & 609 & 296 \\
\hline
\end{tabular}

\section{Schonende Unterzuteilungen}

Auch bei den Unterzuteilungen an die Landeslisten der einzelnen Parteien bleibt im Regelfall alles beim Alten, nur im Ausnahmefall kommen die Sonderregelungen der schonenden Verbindung zum Tragen.

\section{a) Regelfall}

Der Regelfall ist wieder darin zu sehen, dass alle Wahlkreissieger von den Verhältnissitzen getragen werden. Dies trifft insbesondere auf die kleineren Parteien zu, die keine oder nur wenige Wahlkreissieger stellen. Hier unterscheidet sich die schonende Verbindung nicht von dem, was das geltende Recht vorschreibt.

\section{b) Ausnahmefall}

Der Ausnahmefall tritt ein, wenn eine Partei existiert, für die in einem Bundesland die Zahl der Wahlkreissieger die Zahl der Verhältnissitze für ihre Landesliste übersteigt. In einem solchen Fall werden die Wahlkreissieger dieser Partei in diesem Bundesland nach fallenden Erststimmen gereiht. Von den erststimmenstärkeren Wahlkreissiegern bekommen die meisten ein Mandat, nämlich so viele, wie die Zahl der Verhältnissitze vorgibt. Diese Mandate werden sowohl unter den Bedingungen der Mehrheitswahl wie auch unter Anrechnung auf die Landeslisten vergeben. In den wenigen verbleibenden, durch die Unterschiedszahl bestimmten Ausnahmewahlkreisen mit erststimmenschwächeren Wahlkreissiegern wird geprüft, ob Mitbewerber anderer Parteien in den Bundestag einziehen. Falls es einen erfolgreichen Mitbewerber gibt, hat der Wahlkreissieger das Nachsehen und bekommt kein Mandat.

Schließlich ist auch der Fall möglich, dass in einem Ausnahmewahlkreis kein Mitbewerber erfolgreich ist. Hier erhalten die Wahlkreissieger ein Überhangmandat neuer Art. Die Bundestagsgröße wächst um die Zahl dieser Mandate. Auch diese Zusatzman- 
date sind nicht ausschließlich unter den Bedingungen der Mehrheitswahl vergeben, sondern werden wesentlich durch das Prinzip regionaler Repräsentation gerechtfertigt. Im Gegensatz zu Überhangmandaten alter Art haben Überhangmandate neuer Art eine ganz andere Qualität. ${ }^{24}$

\begin{tabular}{|l|cc|c|c|}
\hline \multicolumn{5}{|c|}{ Tabelle 4: Schonende Verbindung 2009 für 609 Sitze, } \\
CDU-Überhangländer mit insgesamt zwanzig Ausnahmewahlkreisen
\end{tabular}

24 Wegen der andersgearteten Qualität und der Identifizierbarkeit der Überhangmandate neuer Art wird das Nachrückerverbot in Überhangländern aus BVerfGE 97 (1998) 317-331 obsolet. 


\begin{tabular}{|lc|cc|c|}
\hline \multicolumn{5}{|c|}{$\begin{array}{c}\text { Tabelle 5: Schonende Verbindung 2009 für 609 Sitze, } \\
\text { Unterzuteilungsrechnung für die CDU-Landeslisten }\end{array}$} \\
\hline Landesliste & Zweitstimmen & $\frac{\text { Zweitstimmen }}{66800}$ & $\begin{array}{c}\text { Verhältnis- } \\
\text { sitze }\end{array}$ & $\begin{array}{c}\text { Überhang- } \\
\text { mandate }\end{array}$ \\
\hline Schleswig-Holstein & 518457 & 7.8 & 8 & 1 \\
Mecklenburg-Vorpommern & 287481 & 4.3 & 4 & 1 \\
Hamburg & 246667 & 3.7 & 4 & \\
Niedersachsen & 1471530 & 22.0 & 22 & \\
Bremen & 80964 & 1.2 & 1 & \\
\hline Brandenburg & 327454 & 4.9 & 5 & \\
Sachsen-Anhalt & 362311 & 5.4 & 5 & \\
Berlin & 393180 & 5.9 & 6 & \\
Nordrhein-Westfalen & 3111478 & 46.6 & 47 & \\
Sachsen & 800898 & 12.0 & 12 & 1 \\
\hline Hessen & 1022822 & 15.3 & 15 & \\
Thüringen & 383778 & 5.7 & 6 & 1 \\
Rheinland-Pfalz & 767487 & 11.49 & 11 & \\
Baden-Württemberg & 1874481 & 28.1 & 28 & 3 \\
Saarland & 179289 & 2.7 & 3 & 7 \\
\hline Summe & 11828277 & & 177 & \\
& Auf je 66 800 Zweitstimmen entfällt rund ein Verhältnissitz. & \\
\hline
\end{tabular}

c) Illustration am Beispiel der Wahl 2009

Von den Unterzuteilungen 2009 fallen vier unter den Regelfall, dass alle Wahlkreissieger von den Verhältnissitzen getragen werden, nämlich die Rechnungen für SPD, FDP, Linke und Grüne. Die CDU stellt einen Ausnahmefall dar.

Wie in Tabelle 3 vorgerechnet, entfallen von 609 Gesamtsitzen 177 Sitze auf die CDU. Werden diese im Verhältnis der Zweitstimmen den fünfzehn CDU-Landeslisten zugeteilt, kommt es in sieben Ländern zu insgesamt zwanzig Ausnahmewahlkreisen. ${ }^{25}$ In jedem dieser Länder werden die Wahlkreissieger nach Erststimmen gereiht, um die Ausnahmewahlkreise zu identifizieren. In Tabelle 4 sind pro Überhangland die jeweiligen Ausnahmewahlkreise aufgelistet.

Aus der dritten Spalte in Tabelle 4 ist ersichtlich, dass in sieben der zwanzig Ausnahmewahlkreise kein Mitbewerber erfolgreich war. Dort werden für die Wahlkreissieger Überhangmandate neuer Art geschaffen. Die anderen dreizehn Ausnahmewahlkreise sind durch Abgeordnete vertreten, die über ihre Landeslisten ein Mandat bekommen. Tabelle 5 fasst die Endrechnung für die CDU-Landeslisten zusammen. Insgesamt enden die Ober- und Unterzuteilungen damit, dass der Bundestag von 598 (Ausgangssitze) über 609 (Oberzuteilungssitze) auf 616 Sitze (zuzüglich sieben Überhangmandate neuer Art) anwächst.

25 Das sind die 21 Überhangmandate alter Art mit der einzigen Änderung, dass in Baden-Württemberg bei 177 zu verteilenden Sitzen nur neun Ausnahmewahlkreise erzeugt werden und nicht wie bei 173 Sitzen zehn. 


\section{Vorteile der schonenden Verbindung}

Aus der Sicht der Wähler ist der Umstand, dass ein Wahlkreis bei der schonenden Verbindung nicht immer durch den Wahlkreissieger vertreten wird, wohl ebenso neu wie aus der Sicht der Bewerber der Umstand, dass ein Wahlkreissieg nicht mehr sicher zu einem Mandat führt. Doch diesem eher als Änderung denn als Nachteil zu empfindenden Wandel stehen vielfältige Vorteile entgegen, die mit der schonenden Verbindung einhergehen.

Erstens lassen sich Nachwahlen mittels der schonenden Verbindung durch eine Ersatzbewerberregelung vermeiden (1). Dadurch wird einer Operationalisierung negativer Stimmgewichte wie 2005 bei der Nachwahl im Wahlkreis 160 (Dresden I) der Wind aus den Segeln genommen. Zweitens werden die Überhangmandate neuer Art auf etwa ein Drittel der Überhangmandate alter Art reduziert (2). Die verbleibenden Überhangmandate dienen drittens der Gewährleistung des Vertretungsanspruchs der Wähler in den Wahlkreisen (3).

Des Weiteren fördert die schonende Verbindung den Wettbewerbscharakter der Wahl. Sie motiviert alle Beteiligte — Wähler, Bewerber und Parteien — darauf hinzuwirken, dass viertens die Wahlkreise möglichst gleich groß zugeschnitten werden (4). Fünftens sind die nicht von Überhangmandaten profitierenden kleineren Parteien eingeladen, gestaltend mitzuwirken. Sie können Überhangmandate verhindern, indem sie für prospektive Ausnahmewahlkreise Bewerber finden, die über die Liste ein Mandat erhalten und dadurch dem Wahlkreissieger das Nachsehen geben (5). Sechstens dürfte die schonende Verbindung auch der Transparenz im Wählerverhalten förderlich sein (6). Schließlich ließe sich die schonende Verbindung notfalls zur Option W verschärfen, die zwar negative Stimmgewichte ausmerzt, aber die genannten Vorteile nicht mehr in demselben Umfang realisiert (7).

\section{Vermeidung von Nachwahlen}

Mit der schonenden Verbindung können Nachwahlen wegen Ausscheidens eines Wahlkreisbewerbers mittels Rückgriff auf Listenkandidaten umgangen werden. Jede zeitlich versetzte Nachwahl bringt eine massive Beeinträchtigung der Wahlgleichheit mit sich, unabhängig vom Auftreten negativer Stimmgewichte. Vier der sieben Nachwahlen seit 1961 mussten wie 2005 im Wahlkreis 160 (Dresden I) einige Wochen nach der Hauptwahl stattfinden. ${ }^{26}$

Als Problemlösung kann $\S 24$ BWahlG die Verbindung von Personen- und Verhältniswahl dahingehend ausnutzen, dass sie das Nachrücken eines Ersatzbewerbers vorsieht. Im geltenden Recht würde dies auf Grund der Eigenständigkeit der Personenwahl als Fremdkörper wirken. Vor dem Hintergrund der schonenden Verbindung könnte dagegen an die Stelle eines verstorbenen Wahlkreisbewerbers derjenige Kandidat aus der

26 Michael Frank Feldkamp, Nachwahlen, Wissenschaftliche Dienste des Deutschen Bundestages, Der aktuelle Begriff Nr. 63/05, 9. September 2005. - Sechs der sieben Nachwahlen seit 1961 hatten ihre Ursache im Tod eines Wahlkreisbewerbers, dabei konnte zweimal die Nachwahl auf den Tag der Hauptwahl gelegt werden. Die siebte Nachwahl wurde durch die Zerstörung eines Wahllokals verursacht. 
Landesliste seiner Partei eintreten, der in der Listenreihenfolge als erster ohne Wahlkreisbewerbung geführt wird. Dies wäre eine pragmatische Lösung eines diffizilen Sonderfalls, andere sind denkbar. ${ }^{27}$

\section{Reduktion der Überhangmandate}

Derzeit sind 97 der 299 Wahlkreise allein durch Wahlkreissieger vertreten. In den übrigen 202 Wahlkreisen sind auch Listenkandidaten gewählt. Dementsprechend wird allgemein grob ein Drittel der Wahlkreise allein durch den Wahlkreissieger vertreten sein und für sie Überhangmandate neuer Art nötig machen. Beim Übergang vom geltenden Recht zur schonenden Verbindung wird somit etwa ein Drittel der Überhangmandate alter Art fortdauern. ${ }^{28}$

Ein kumulativer Rückblick bestätigt diese Überschlagsrechnung. In den Bundestagswahlen von 1980 bis 2005 kommen in der Summe sechzig Überhangmandate zusammen, von denen gemäß schonender Verbindung achtzehn als Überhangmandate neuer Art fortbestünden.

\section{Vertretungsanspruch der Wahlkreise}

Die schonende Verbindung stellt sicher, dass aus jedem Wahlkreis mindestens einer der Bewerber als Abgeordneter in den Bundestag gewählt wird. Das geltende Recht bezieht diesen Wahlakt ausschließlich auf das Erststimmenergebnis, indem das Direktmandat automatisch an den Wahlkreissieger fällt. Im Gegensatz dazu nimmt die schonende Verbindung auf das im Bundeswahlgesetz formulierte Ziel Bezug, die Personenwahl mit der Verhältniswahl zu verbinden. Wir interpretieren $\S 5$ BWahlG dahingehend, dass nicht nur der mittels Erststimmenerfolg gekürte Wahlkreissieger den Wahlkreis vertreten kann, sondern auch ein mittels Zweitstimmenanteil über die Landesliste einrückender Mitbewerber.

Bei streng juristischer Betrachtung geht damit in gewisser Weise die Unmittelbarkeit verloren, die für die Erststimme typisch ist, dass nämlich die Wähler unmittelbar über die personelle Vertretung ihres Wahlkreises im Bundestag entscheiden. Bei faktischer Betrachtung dagegen ist die Wahl von vornherein auf Kandidaten beschränkt, die die Parteien in Kreiswahlvorschlägen aufgestellt haben. Im Zweistimmenwahlsystem wird

27 Mit der Ersatzbewerberregelung hätte 2005 nach dem Tod der NPD-Bewerberin im Wahlkreis 160 (Dresden I) der erste Kandidat aus der NPD-Landesliste ohne Wahlkreisbewerbung die Bewerbung übernommen. Dies wäre Platz 2 der sächsischen NPD-Liste gewesen, ein Kandidat aus Coburg. Auf Platz 8 wurde ein Kandidat aus Dresden geführt, der sich ebenfalls in keinem Wahlkreis beworben hatte (Wir danken Renate Recknagel vom Statistischen Landesamt des Freistaates Sachsen für die Bereitstellung der Liste der sächsischen Wahlkreisbewerber.). Die Wahl hätte am Tage der Hauptwahl stattfinden können. Die Wähler in Dresden wären negativen Stimmgewichten nicht anders ausgesetzt gewesen als die anderen Wähler in Sachsen.

28 Friedrich Pukelsheim/Matthias Rossi, JuristenZeitung 65 (2010) 922-927. Sophie-Charlotte Lenski, Paradoxien der personalisierten Verhältniswahl, Archiv des öffentlichen Rechts 134 (2009) 473-512 (504). 
mit der Erststimme zwar demonstrativ für alle Beteiligten sichtbar, dass die lokale Bindung zwischen Wählern und Abgeordneten ein wesentlicher Aspekt ist, der die Stellung der Abgeordneten legitimiert. Aber auch Einzelbewerber und Abgeordnete, die über die Listen ein Mandat erhalten, betreiben in der Regel ein Wahlkreisbüro und agieren als Vertreter ihres Wahlkreises. ${ }^{29}$

\section{Gleichheit der Wahlkreise}

Da bei der schonenden Verbindung im Ausnahmefall durch die Reihung der Wahlkreissieger nach absoluten Erststimmen das Reservoir an potentiellen Wählern mitentscheidend sein kann, erhöht sich die Motivation für die Entscheidungsträger, möglichst gleich große Wahlkreise einzurichten, die auch aus Wählersicht erstrebenswert sind.

Zum Beispiel reichen 2009 die zwanzig Ausnahmewahlkreise in den Tabellen 2 und 4 vom bundesweit kleinsten Wahlkreis 227 (Deggendorf) mit 189600 Einwohnern bis zum 149. kleinsten Wahlkreis 192 (Gotha/Ilm-Kreis) mit 248381 Einwohnern. Alle Ausnahmewahlkreise gehören also zur Hälfte der kleineren Wahlkreise. Sechs Ausnahmewahlkreise zählen sogar zu den sehr kleinen Wahlkreisen mit weniger als 212676 Einwohnern, die vom Durchschnitt 250222.4 um mehr als fünfzehn Prozent nach unten abweichen. ${ }^{30}$

Der Anreiz, gleich große Wahlkreise einzurichten, kommt nur mit den (absoluten) Erststimmenzahlen zu Stande, nicht mit den (prozentualen) Erststimmenanteilen. Der Sieger im Wahlkreis 227 (Deggendorf) hat 2009 mit 53 \% mehr als die Hälfte der Erststimmen auf sich vereinigt. Dieses Ergebnis verliert an Aura, wenn man bemerkt, dass

29 Jedes Mitglied des Bundestages bekommt die Kosten für ein Wahlkreisbüro erstattet (§ 12 II 1 AbgG). - In der 1996 tagende Reformkommission zur Größe des Deutschen Bundestages stand die Frage, ob Wahlkreisbewerber, die über ihre Landesliste gewählt sind, ebenfalls den Wahlkreis vertreten können, nicht zur Debatte. In den erstatteten Rechtsgutachten lässt jedoch nichts darauf schließen, dass ausschließlich Wahlkreissieger befähigt wären, die Wähler des Wahlkreises im Bundestag zu repräsentieren. Siehe Markus Heintzen, Rechtsgutachten zu Fragen des Bundestagswahlrechts im Hinblick auf die Wahl zum 14. Deutschen Bundestag, Reformkommission zur Größe des Deutschen Bundestages, Drucksache 9, Halle, 1996. Wolfgang Löwer, Aktuelle wahlrechtliche Verfassungsfragen, Rechtsgutachten erstattet dem Deutschen Bundestag, Bonn, 1997.

30 Es ist $74816496 / 299=250222.4$ (Wir danken Manfred Thoma vom Statistischen Bundesamt für die Bereitstellung der Bevölkerungszahlen zum Stichtag 31. Dezember 2008.). Andere Länder orientieren die Wahlkreiseinteilung an der Zahl der Wahlberechtigten, siehe das Gutachten des Max-Planck-Instituts für ausländisches öffentliches Recht und Völkerrecht, Wahlkreiseinteilung in westlichen europäischen Demokratien, den USA und Kanada, Zeitschrift für ausländisches öffentliches Recht und Völkerrecht - Heidelberg Journal of International Law 57 (1997) 633-674. — Für die Wahl 2009 legte die Wahlkreiskommission zwei Berichte vor (Bundestagsdrucksachen 16/4300 und 16/6286, mit Bevölkerungszahlen zum 31. Dezember 2006). Die vom Bundestag am 24. Januar 2008 beschlossene Wahlkreiseinteilung weicht davon ab. Weder in den Beratungen im Parlament noch in den begleitenden Drucksachen wurden die Abweichungen begründet und die neuen Wahlkreisgrößen dokumentiert. - Ein Beispiel eklatant ungleicher Wahlkreisgeometrie analysiert Hans Herbert von Arnim, Der Zuschnitt der hessischen Wahlkreise ist verfassungswidrig, Deutsches Verwaltungsblatt 118 (2003) 553-624. 
im kleinsten Wahlkreis der Republik die Wählerschaft homogener ausfallen kann als in anderen. ${ }^{31}$

\section{Stärkung des politischen Wettbewerbs}

Die schonende Verbindung eröffnet auch kleineren Parteien die Möglichkeit, zur Verhinderung von Überhangmandaten beizutragen. Dies wird beispielhaft bei der Wahl 2009 deutlich. In sechzehn Ausnahmewahlkreisen kommt es nicht zu Überhangmandaten, weil die Wahlkreise durch Wahlkreisbewerber vertreten werden, die über die Landeslisten in den Bundestag einziehen. Parteien, die nicht durch Überhangmandate begünstigt sind, sollten demnach ihre Kandidaten auf aussichtsreichen Listenplätzen dazu bewegen, sich in vermuteten Ausnahmewahlkreisen zu bewerben. Gemäß Abschnitt V.4 reicht es, sich auf die etwa 150 Wahlkreise zu konzentrieren, die kleiner sind als der Durchschnitt. Bestätigt sich die Vermutung und kommen die Mitbewerber zum Zuge, haben die Wahlkreissieger das Nachsehen und Überhangmandate werden umgangen. ${ }^{32}$

Aus Sicht der Wahlkreisbewerber schafft die schonende Verbindung eine Legitimation, die über den Wahlkreis hinausreicht. Ein Wahlkreisbewerber tritt nicht mehr nur im Wahlkreis gegen die Mitbewerber aus anderen Parteien an, sondern er konkurriert auch mit den Parteifreunden der eigenen Partei, die sich in anderen Wahlkreisen des Landes bewerben. Um ein Mandat zu erhalten, muss er in der Reihung der Erststimmen weit genug vorne stehen. Für die Erststimmenreihung haben die Wähler das letzte Wort, wie es das demokratische Prinzip gemäß Art. 20 Abs. 2 Satz 2 GG verlangt. Dagegen ist die Nominierung auf der Landesliste eine parteiinterne Angelegenheit, die wegen der verfassungsrechtlich abgesicherten Mitwirkung der Parteien an der politischen Willensbildung freilich ebenso zulässig ist. ${ }^{33}$

31 Als Mitglied der Europäischen Kommission für Demokratie und Recht (Venedig-Kommission) empfiehlt Deutschland seit 2002 auf internationalem Parkett, bei der Wahlkreiseinteilung Höchstabweichungen von $10 \%$ und $15 \%$ einzuhalten [www.venice.coe.int/docs/2002/ CDL-AD(2002)023rev-ger.pdf]. Im nationalen Raum dauern derweil die Abweichungsschwellen $15 \%$ und $25 \%$ unberührt fort ( 33 III 3 BWahlG). - In Pennsylvania umfasst aktuell bei den Wahlen zum US-Repräsentantenhaus jeder der 19 Wahlkreise entweder 646371 oder 646372 Einwohner, in Texas jeder der 32 Wahlkreise entweder 651619 oder 651620 Einwohner. Siehe Michel Balinski, Fair Majority Voting (or How to Eliminate Gerrymandering), American Mathematical Monthly 115 (2008) 97-113.

$32 \mathrm{Im}$ amtierenden Bundestag haben dreizehn Abgeordnete sich nicht in einem Wahlkreis beworben, und zwar acht von der Linken (SH, HH, NI, HB, NW, TH, $2 \times \mathrm{SL}$ ), je zwei von SPD (HH, NW) und FDP (HH, BE), sowie einer von den Grünen (SL).

33 Heiko Franke/Andreas Grimmel, Wahlen mit System? Reformüberlegungen zur personalisierten Verhältniswahl, Zeitschrift für Parlamentsfragen 38 (2007) 591-602. Philipp Manow, Wiederwahlwahrscheinlichkeiten im deutschen System der personalisierten Verhältniswahl - Eine empirische Untersuchung der 16 Bundestagswahlen, 1949-2005, Zeitschrift für Politikwissenschaft 18 (2008) 147-166. Philip Manow/Martina Nistor, Wann ist ein Listenplatz sicher? Eine Untersuchung der Bundestagswahlen 1953 bis 2002, Zeitschrift für Parlamentsfragen 40 (2009) 603-620. Suzanne Shirley Schüttemeyer/Roland Sturm, Der Kandidat - das (fast) unbekannte Wesen: Befunde und Überlegungen zur Aufstellung der Bewerber zum Deutschen Bundestag, Zeitschrift für Parlamentsfragen 36 (2005) 539-553. 


\section{Transparenz der Stimmenwirkung}

Die schonende Verbindung stärkt die Wirkung von Erst- und Zweitstimmen so, wie die beiden Stimmen im Wahlsystem wirken sollen. Wenn beispielsweise in baden-württembergischen Wahlkreisen viele Wähler mit ihrer Erststimme den CDU-Wahlkreisbewerber wählen und mit ihrer Zweitstimme die FDP-Landesliste, verhelfen sie dem CDU-Sieger zu einem vorderen Rang in der Erststimmenreihung und damit eher zu einem Mandat. Mit einem solchem Stimmensplitting haben die Wähler genau den Effekt erzielt, der mit der Erststimme erzielt werden soll, sie unterstützen den Wahlkreissieger als Person.

Andererseits fällt die verhältnismäßige Umgewichtung durch Stimmensplitting bei der schonenden Verbindung geringer aus als beim geltenden Recht. Diejenigen Wahlkreissieger, die weniger Erststimmen erhalten, müssen in Ausnahmewahlkreisen gegebenenfalls erfolgreichen Mitbewerbern den Vortritt lassen. Stimmensplitting übt eine abträgliche Fernwirkung auf andere Wahlkreise aus, deren Sieger wegen zu schwacher Erststimmen zurückbleiben. ${ }^{34}$

\section{Option $W$ - Wahlkreisrepräsentation und Verhältnismäßigkeit}

Die schonende Verbindung lässt die Möglichkeit bestehen, dass während einer der Unterzuteilungen Überhangmandate neuer Art anfallen können, die auch die Möglichkeit negativer Stimmgewichte implizieren. Will man auch diese Restwahrscheinlichkeit radikal ausschalten, bietet sich eine Variante an, die wir Option W nennen, da auch sie die Wahlkreisrepräsentation garantiert.

Option W befolgt in der Oberzuteilung und in den Unterzuteilungen die strikte Regel, in Ausnahmefällen so viele erststimmenschwächere und mit erfolgreichen Mitbewerbern flankierte Wahlkreissieger mandatslos zurückzulassen, dass keine Überhänge entstehen.

Im Endergebnis gewährleistet Option W das Prinzip der Wahlkreisrepräsentation, weil Wahlkreissieger ohne erfolgreiche Mitbewerber nicht zur Disposition gestellt werden. Das Verfahren ist weniger aufwändig, da Ober- und Unterzuteilungen nur einmal berechnet werden und die Mandatsvergabe an die Wahlkreissieger dem Ergebnis der Verhältnisrechnungen unterworfen wird. Die vorgegebene Gesamtzahl von 598 Sitzen wird genau eingehalten.

Wendet man Option W auf die Wahl von 2009 an, so erhält man als Oberzuteilung das, was in Tabelle 1 als Anfangsrechnung für die schonende Verbindung ausgewiesen

34 Ossip Fürnberg/Danko Knothe, Wahlsieg ohne Stimmenmehrheit: Auswirkungen von verstärktem "Lagersplitting" auf Mandatsverteilung und Koalitionsoptionen, Zeitschrift für Parlamentsfragen 40 (2009) 56-74. Thomas Gschwend, Ticket-splitting and strategic voting in mixed electoral systems, European Journal of Political Research 46 (2007) 1-23. Eckhard Jesse, Split-voting in the Federal Republic of Germany: An analysis of the Federal Elections from 1953 to 1987, Electoral Studies 7 (1988) 109-124. Harald Schoen, Split-ticket voting in German Federal elections, 1953-90: An example of sophisticated balloting? Electoral Studies 18 (1999) 473-496. Markus Winkler, Gleichheit der Wahl, "Stimmensplitting" und Überhangmandate, Recht und Politik - Vierteljahreshefte für Rechts- und Verwaltungspolitik 35 (1999) 244-247. 
ist. Für die 45 CSU-Wahlkreissieger stellt die Verhältnisrechnung nur 42 Sitze bereit. Das Nachsehen haben die Sieger im Wahlkreis 229 (Passau), im Wahlkreis 244 (Nürnberg-Nord) und im Wahlkreis 245 (Nürnberg-Süd). Dagegen kommt im Wahlkreis 227 (Deggendorf) der Wahlkreissieger zum Zug, da ihm kein erfolgreicher Mitbewerber den Platz streitig macht. ${ }^{35}$ Die Unterzuteilung der CDU-Sitze an die CDU-Landeslisten erfordert eine analoge Prüfung.

\section{Vorschlag für Änderung des BWahlG}

Die schonende Verbindung von Personen- und Verhältniswahl bedarf der sorgfältigen und präzisen Verankerung im BWahlG. Wir unterbreiten nachfolgend einen Vorschlag für entsprechende Formulierungen. Änderungen gegenüber dem geltenden BWahlG sind kursiv gesetzt, Erläuterungen in kleiner Schrift angefügt.

\section{$\S 5$ Wahl in den Wahlkreisen}

(1) ${ }^{1}$ Aus jedem Wahlkreis wird mindestens einer der Wahlkreisbewerber als Abgeordneter gewählt. ${ }^{2}$ Wahlkreissieger ist der Bewerber, der die meisten Stimmen auf sich vereinigt. ${ }^{3}$ Bei Stimmengleichheit entscheidet das vom Kreiswahlleiter zu ziehende Los. Die Feststellung des Wahlkreissiegers wird ein eigener Schritt und ist von der im Folgenden geregelten Zuteilung eines Mandats getrennt. Im Regelfall erhält der Wahlkreissieger ein Mandat, im Ausnahmefall wird der Wahlkreis durch einen (oder mehrere) Mitbewerber einer anderen Partei vertreten.

(2) Der Wahlkreissieger erhält ein Mandat,

1. wenn ihm unter allen Wahlkreissiegern seiner Partei in seinem Land in der Reihung nach fallenden Erststimmen ein Rang zukommt, der die Zahl der nach \& 7 Absatz 1 der Landesliste seiner Partei zugeteilten Sitze nicht übertrifft,

2. oder wenn in seinem Wahlkreis kein anderer Bewerber einen Sitz aus einer Landesliste erhält,

Wenn $§ 7$ Absatz 1 der Landesliste einer Partei beispielsweise zehn Sitze zuteilt, so bekommen bis zu zehn Wahlkreissieger dieser Partei in diesem Land ein Mandat. Im Fall von mehr als zehn Wahlkreissiegern werden sie gemäß fallender Erststimmenzahlen gereiht. Die ersten zehn erhalten ein Mandat. Jenseits der ersten zehn bekommen Wahlkreissieger nur dann ein Mandat, falls kein Mitbewerber einer anderen Partei über die Landesliste in den Bundestag einzieht. Damit wird Absatz 1 Genüge getan, dass aus jedem Wahlkreis mindestens ein Abgeordneter gewählt ist.

3. oder wenn er von Wahlberechtigten gemä $\beta \S 20$ Absatz 3 oder von einer Partei vorgeschlagen ist, für die in dem betreffenden Land keine Landesliste zugelassen ist oder auf deren Landesliste keine zuteilungsberechtigten Zweitstimmen entfallen. Die Gesamtzahl der Sitze nach $\$ 1$ Absatz 1 wird um die Zahl der in diesem 3. Fall zugeteilten Sitze vermindert.

35 Der Sieger im Wahlkreis 229 (Passau) steht auf Rang 42 mit 54275 Erststimmen $=47 \%$, der Wahlkreis ist auch vertreten durch einen Abgeordneten der FDP. Für die anderen genannten Wahlkreise siehe Tabelle 2. 
Die Regelung betrifft Bewerber, die parteilos sind oder deren Partei mangels Landesliste oder mangels zuteilungsberechtigter Zweitstimmen an der Verhältnisrechnung nicht teilnimmt. Dies ist bisher im geltenden $\S 6$ Absatz 1 mit Verweis auf $\S 6$ Absatz 6 geregelt.

\section{$\S 6$ Oberzuteilung an die Parteien auf Bundesebene}

Der geltende $\S 6$ (Wahl nach Landeslisten) heißt hier „Oberzuteilung an die Parteien auf Bundesebene", $\S 7$ (Listenverbindung) "Unterzuteilungen an die Landeslisten der Parteien".

(1) Eine Zweitstimme ist zuteilungsberechtigt, sofern sie gültig ist und auf eine Partei entfällt, die mindestens fünf Prozent der gültigen Zweitstimmen erhält oder mindestens drei Wahlkreissieger stellt oder eine nationale Minderheit vertritt.

Dies ist inhaltsgleich mit dem geltenden $\S 6$ Absatz 6. Der „Doppelerfolgsausschluss" aus dem geltenden $\S 6$ Absatz 1 Satz 2 ist ersatzlos gestrichen. Die schonende Verbindung von Personenwahl und Verhältniswahl soll alle Wähler zu einem aus ihrer Sicht bestmöglichen Gebrauch beider Stimmen einladen, ohne dass vom Gesetz erlaubte Eigenwilligkeiten im Nachhinein mit der Wegnahme der Zweitstimme geahndet werden.

(2) ${ }^{1}$ Für jede Partei werden die zuteilungsberechtigten Zweitstimmen im Bundesgebiet zusammengezählt. 'Diese Stimmensummen werden durch einen Bundesdivisor geteilt. ${ }^{3} J e d e s$ Teilungsergebnis wird zur nächstgelegenen ganzen Zahl gerundet, die bestimmt, wie viele Sitze auf die Partei insgesamt entfallen. 4Der Bundesdivisor ist so zu bestimmen, dass alle nach $\$ 5$ Absatz 2 Satz 3 verbleibenden Sitze zugeteilt werden. 5Zur Berechnung des Bundesdivisors wird die Gesamtzahl der zuteilungsberechtigten Stimmen durch die Gesamtzahl der verfügbaren Sitze geteilt, das Ergebnis ist der Ausgangswert für den Zuteilungsdivisor. 6Werden mit diesem Ausgangswert mehr Sitze vergeben als verfügbar, ist der Zuteilungsdivisor geeignet heraufzusetzen; werden weniger vergeben, ist er geeignet herabzusetzen.

Diese Regelung bestimmt als Sitzzuteilungsmethode für die Parteien das Divisorverfahren mit Standardrundung (Sainte-Laguë/Schepers). Damit wird dem Grundsatz der Erfolgswertgleichheit der Wählerstimmen bundesweit in einer bestmöglichen Weise Genüge getan. Die Vorschrift zur Divisorbestimmung ist der geltende $\S 6$ Absatz 2 Satz 5-7.

(3) Falls für eine oder mehrere Parteien die nach Absatz 2 bestimmte Zahl ihrer Sitze insgesamt kleiner ist als die bundesweite Zahl der Mandate für ihre Wahlkreissieger nach $\S 5$ Absatz 2, wird die nach $\$ 5$ Absatz 2 Satz 3 verbleibende Zahl der Sitze in Einerschritten erhöht, bis die erneute Berechnung nach $\$ 5$ Absatz 2, § 6 Absatz 2 und $\S 7$ Absatz 1 für jede Partei allen Landeslisten eine Sitzzahl zuteilt, die in jedem Land der Zahl der Mandate für ihre dortigen Wahlkreissieger nach § 5 Absatz 2 gleichkommt oder sie übertrifft.

Um in der Oberzuteilung keine Überhangmandate entstehen zu lassen, wird die Bundestagsgröße erhöht, bis alle Wahlkreissieger von den Verhältnissitzen getragen sind. Bei mehreren überhanggefährdeten Parteien können einige Parteien mit ihren endgültigen Sitzzahlen die Zahl der Wahlkreissieger übertreffen.

(4) Ergeben sich dabei mehrere mögliche Sitzzuteilungen, so entscheidet das vom Bundeswahlleiter zu ziehende Los.

Die Pattauflösung ist gleichlautend mit der zweiten Hälfte von Satz 4 im geltenden $\S 6$ Absatz 2.

(5) Erhält eine Partei, auf die mehr als die Hälfte aller zuteilungsberechtigten Zweitstimmen entfällt, nicht mehr als die Hälfte aller Bundestagssitze, so werden für sie so viele weitere Sitze geschaffen, bis sie über eine absolute Sitzmehrheit verfügt. 
Diese Mehrheitsklausel verbessert die reparaturbedürftige Fassung des geltenden $\S 6$ Absatz 3. Bislang gibt es keine Bundestagswahl, bei der eine Mehrheitsklausel Anwendung gefunden hätte.

\section{$\S 7$ Unterzuteilungen an die Landeslisten der Parteien}

(1) ${ }^{1}$ Für jede Partei werden die zuteilungsberechtigten Zweitstimmen ihrer Landeslisten durch einen Parteidivisor geteilt. 2Jedes Teilungsergebnis wird zur nächstgelegenen ganzen Zahl gerundet, die bestimmt, wie viele Sitze auf die Landesliste entfallen. 3Der Parteidivisor wird so bestimmt, dass die Summe aller Landeslistensitze der Sitzzahl gleichkommt, die auf die Partei insgesamt entfallen.

Diese Vorschrift bestimmt als Sitzzuteilungsmethode für die Landeslisten das Divisorverfahren mit Standardrundung (Sainte-Laguë/Schepers). Damit wird dem Grundsatz der Erfolgswertgleichheit der Wählerstimmen Genüge getan mit der Einschränkung, dass gemäß § 7 Absatz 4 Überhangmandate möglich bleiben.

(2) Ergeben sich dabei mehrere mögliche Sitzzuteilungen, so entscheidet das vom Bundeswahlleiter zu ziehende Los.

Die Pattauflösung ist gleichlautend mit der zweiten Hälfte von Satz 4 im geltenden $§ 6$ Absatz 2.

(3) ${ }^{1}$ Von der für jede Landesliste auf diese Weise ermittelten Zahl der Sitze wird die Zahl der von der Partei in den Wahlkreisen des Landes errungenen Mandate abgerechnet. ${ }^{2}$ Etwaige restliche Sitze werden aus der Landesliste in der dort festgelegten Reihenfolge besetzt. ${ }^{3}$ Bewerber, die in einem Wahlkreis gewählt sind, bleiben auf der Landesliste unberücksichtigt. ${ }^{4}$ Entfallen auf eine Landesliste mehr Sitze als Bewerber benannt sind, so bleiben diese Sitze unbesetzt.

Dies ist der alte $\S 6$ Absatz 4.

(4) ${ }^{1}$ Mandate für Wahlkreissieger gemä $\beta$ 5 Absatz 2 verbleiben einer Landesliste auch dann, wenn sie die nach den Absätzen 1 und 2 ermittelte Sitzzahl übersteigen. ${ }^{2}$ In einem solchen Fall erhöht sich die Gesamtzahl der Sitze nach $\S 1$ Absatz 1 um die Unterschiedszahl; eine erneute Berechnung nach $\S 6$ und $\S 7$ Absatz 1 findet nicht statt. Dies ist im Wesentlichen der geltende $\S 6$ Absatz 5 und definiert die Überhangmandate neuer Art.

\section{§ 24 Änderung von Kreiswahlvorschlägen}

${ }^{1}$ Ein Kreiswahlvorschlag kann nach Ablauf der Einreichungsfrist nur durch gemeinsame schriftliche Erklärung der Vertrauensperson und der stellvertretenden Vertrauensperson und nur dann geändert werden, wenn der Bewerber stirbt oder die Wählbarkeit verliert. ${ }^{2}$ Das Verfahren nach $\$ 21$ braucht nicht eingehalten zu werden, der Unterschriften nach $\S 20$ Absatz 2 und 3 bedarf es nicht. ${ }^{3}$ Nach der Entscheidung über die Zulassung eines Kreiswahlvorschlages (\$26 Absatz 1 Satz 1) tritt an die Stelle eines Wahlkreisbewerbers, der gestorben ist oder die Wählbarkeit verloren hat, der Kandidat aus der Landesliste seiner Partei, der als erster in der Listenreihenfolge sich nicht in einem Wahlkreis bewirbt. ${ }^{4}$ Ist ein solcher nicht verfügbar, entfällt der Kreiswahlvorschlag ersatzlos.

Eine Nachwahl wie 2005 im Wahlkreis 160 (Dresden I) führt immer zu einer massiven Beeinträchtigung des Systems. Es gibt keinen Königsweg, um die so ausgelösten Verwerfungen bruchlos zu heilen. Da die 
Novellierung eine Verbindung zwischen Personenwahl und Verhältniswahl herstellt, erscheint deren Einsatz für die Nachfolge eines verstorbenen Wahlkreiskandidaten vertretbar.

$\S 43$ (Nachwahl) muss dann angepasst werden.

\section{Ausblick}

Die hier in den Mittelpunkt gestellte schonende Verbindung hat zum Ziel, in möglichst großer Nähe zum geltenden Wahlsystem zu bleiben und die reklamierten Unwuchten durch eine ausgewogene Verbindung von Personen- und Verhältniswahl zu beseitigen. Je weiter man sich vom derzeitigen System entfernt, desto vielfältiger werden die Novellierungsmöglichkeiten. Auf der Personenwahlseite könnte man etwa Hürden setzen, dass nur Wahlkreissieger mit mindestens $35 \%$ Erststimmenanteil ein Mandat erhalten. Auf der Verhältniswahlseite könnten Ergänzungsmandate ins Leben gerufen werden, um etwaigen Überhangmandate ein Gegengewicht zu bieten. Dem Erfindungsreichtum sind kaum Grenzen gesetzt. In welche Richtung die politischen Akteure gehen wollen und was sich dann im Bundestag als mehrheitsfähig erweist, bleibt abzuwarten. 